\title{
Reabilitação de redes coletivas de sistemas pressurizados de irrigação
}

\author{
Heber P. Gomes ${ }^{1} \&$ Saulo de T. M. Bezerra \\ 1 Departamento de Engenharia Civil, CT/UFPB. Cidade Universitária, CEP 58050-900, João Pessoa, PB, Brasil. Fone: \\ (83) 3216-7684,E-mail: heber@Irh.ct.ufpb.br (Foto); saulodetarso_civil@yahoo.com.br. \\ Protocolo $124-1 / 8 / 2004$ - Aprovado em 1/6/2005
}

\begin{abstract}
Resumo: Apresenta-se, neste trabalho, um método de otimização econômica para a reabilitação de redes ramificadas pressurizadas de distribuição de água para projetos de irrigação que se encontram com deficiência de vazão e pressão nos pontos de consumo. O método poderá ser aplicado a redes malhadas de abastecimento urbano, desde que sejam transformadas em ramificadas, através do método do seccionamento fictício. A metodologia empregada se baseia no método Granados, de otimização econômica de redes pressurizadas; trata-se de um processo iterativo que seleciona, a cada passo, as possibilidades de modificação dos diâmetros das tubulações da rede, de forma a minimizar o custo de investimento da reabilitação do sistema. O método foi testado para uma rede de distribuição que abastece um projeto de irrigação fictício, no qual existe deficiência de pressão em quase todos os pontos de consumo; enfim, o custo para a reabilitação da rede foi de $41 \%$ do custo original do sistema.
\end{abstract}

Palavras-chave: otimização, irrigação pressurizada, rede de abastecimento

\section{Rehabilitation of collective networks of pressurized irrigation systems}

\begin{abstract}
This paper presents a method of economic optimization aiming the rehabilitation of pressurized branched networks of water distribution for irrigation projects, which have flow and pressure deficiency at the consumption points. The method can be applied to looped networks of urban water supply, on condition that they are changed into branched ones, through the fictitious sectioning method. The methodology used is based on the Granados method of economic optimization of pressurized networks, which is an iterative process that selects step by step the possibilities of changes of the network pipe diameters, so as to optimize the investment cost of the rehabilitation system. The method was tested for a distribution network that supplies a fictitious irrigation project, in which there is pressure deficiency in almost all the consumption points. The network rehabilitation cost was $41 \%$ of the original cost.
\end{abstract}

Key words: optimization, pressurized irrigation, supply network

\section{INTRODUÇÃO}

As redes coletivas pressurizadas de abastecimento de perímetros irrigados compreendem as estruturas hidráulicas de tubulações ramificadas, responsáveis pelo suprimento de água com vazão e pressão suficientes para atender às necessidades hídricas das parcelas irrigadas; além disso, elas constituem uma parte importante dos perímetros irrigados, em função dos custos elevados para a sua implantação, manutenção e operação.

Um dos maiores problemas na operação das redes de distribuição de água com o seu sistema de impulsão é o aumento não previsto da demanda de água ao longo do tempo, que ocorre, normalmente, devido às expansões não previstas nas áreas das parcelas irrigadas, na ampliação do perímetro e por mudanças no uso do solo. Com o crescimento da demanda de água nas parcelas irrigadas, os requerimentos de vazões nos trechos se elevam, provocando maiores perdas de carga nas tubulações. Com o aumento das perdas nas tubulações da rede coletiva, diminuirão as pressões disponíveis nas tomadas de água, prejudicando consideravelmente as parcelas irrigadas, sobretudo aquelas situadas em cotas mais elevadas ou nas extremidades do perímetro. Este problema ocorre na maioria dos perímetros irrigados do mundo. 
Devido ao impasse antes apontado, é notória a necessidade de reabilitar o sistema coletivo de distribuição de água, por meio de intervenções físicas na rede de distribuição, de maneira que a água possa alcançar as parcelas irrigadas com vazões e pressões suficientes. O termo reabilitação é relativamente novo no meio científico, que concentrou seus esforços, durante as últimas décadas, no desenvolvimento de metodologias para o projeto ótimo das redes de distribuição.

O processo de reabilitação de sistemas de abastecimento de água pode ser dividido em duas fases, que são a do diagnóstico e a de implantação de medidas para a superação dos problemas. O diagnóstico do sistema de distribuição compreende o conhecimento da questão, através da simulação do funcionamento hidráulico do abastecimento, com a calibração de modelos que possam reproduzir o que, efetivamente, se dá na prática. Após a obtenção do diagnóstico do problema, deve-se estabelecer estratégias de reabilitação, levando-se em conta os aspectos técnicos e econômicos (minimização de custos).

A reabilitação dos sistemas de distribuição de água é uma prática já consagrada, que vem sendo empregada principalmente nas redes pressurizadas de abastecimento a núcleos urbanos. Muitas reabilitações foram realizadas em civilizações que sofreram conflitos históricos (guerras); no entanto, só se consolidaram como técnica científica com o surgimento das tubulações de ferro fundido (Cheung, 2004). Lam (1973) abordou que sistemas de distribuição de água se tornam inadequados depois de determinado período de tempo e devem ser expandidos (aumentando o diâmetro) ou ampliados (aumentando a rede para abranger novas áreas que não tinham redes originalmente). Swamee \& Sharma (1990) apresentaram um processo chamado Reorganização de Sistemas de Distribuição de Água, abordando a necessidade de reorganizálos em função do aumento da demanda. Se este aumento for pequeno, então a melhoria do bombeamento é, geralmente, suficiente; entretanto, se ele é significativo, deverão ser introduzidas tubulações em paralelo, substituir tubulações, aumentar a capacidade de bombeamento e/ou instalar novas estações de recalque.

Em geral, as técnicas usadas na reabilitação da maioria das redes hidráulicas implantadas no mundo se baseiam em métodos de tentativa e erro. Este tipo de metodologia faz tão somente o balanceamento hidráulico do sistema, sem levar em consideração a busca de uma solução que minimize os custos de implantação das novas tubulações da rede. Dentre os métodos específicos para análises de reabilitação baseados em técnicas estatísticas, pode-se destacar os trabalhos desenvolvidos por Shamir \& Howard (1979); Walski \& Pelliccia (1982) e Le Gat \& Einsenbeis (2000). Segundo Cheung (2004) diversas aplicações utilizando-se métodos clássicos de otimização vêm sendo propostas na literatura (Li \& Haimes, 1992; Kim \& Mays, 1994; Kleiner et al., 1998) para auxiliar na escolha das melhores opções de reabilitação. Pelos modelos baseados na metodologia de algoritmos genéticos, pode-se citar: Murphy \& Simpson (1992); Halhal et al. (1997); De Schanetzen et al. (1998); Walters et al. (1999); Dandy \& Engelhardt (2001) e Cheung (2004).

Objetivou-se, com este trabalho, apresentar um método de otimização econômica para a reabilitação de redes ramificadas pressurizadas de distribuição de água destinadas a projetos de irrigação, que se encontram com deficiência de vazão e pressão nos pontos de consumo.

\section{METODOLOGIA}

A metodologia empregada neste trabalho está baseada no método Granados (1990) e se divide em duas etapas, em que a primeira consiste na determinação da solução inicial, na qual a rede será composta por seus diâmetros originais, submetidos às novas vazões de projeto. Em conseqüência do aumento das vazões ocorrem perdas de carga maiores que as consideradas no projeto original, fazendo com que o sistema requeira uma pressão maior de distribuição; esta pressão, necessária para alimentar a rede com as novas demandas do projeto, será representada por uma cota de cabeceira fictícia, que é uma condição de contorno do método; já a segunda etapa compreende uma seqüência de iterações, na qual se diminui paulatinamente (a partir da solução inicial) a cota piezométrica de alimentação e se obtém, para cada decréscimo desta cota, um novo custo de reabilitação da rede de distribuição, decorrente da substituição das tubulações por outras, de maiores diâmetros. O processo iterativo termina quando se alcança a cota de cabeceira fixa do projeto original.

O algoritmo utilizado para a determinação da solução inicial pode ser sintetizado:

- Cálculo da carga hidráulica fictícia (CHf) de cada nó, que é igual à cota do terreno, mais as perdas de carga nos trechos à montante do nó, mais a pressão requerida neste ponto:

$$
\mathrm{CHf}=\mathrm{Ct}+\sum \mathrm{Hf}_{\text {mon }}+\text { Preq }
$$

em que:

CHf - carga hidráulica fictícia do nó, em m

$\mathrm{Ct}$ - cota do terreno do nó, em $\mathrm{m}$

$\sum \mathrm{Hf}_{\text {mon }}$ - somatório das perdas de carga nos trechos a montante do nó, em m

Preq - pressão requerida no nó, em m

- Determinação da cota de cabeceira fictícia inicial, que corresponderá ao valor da carga hidráulica fictícia máxima $\left(\mathrm{CHf}_{\max }\right)$ :

$$
\mathrm{Zo}=\mathrm{CHf}_{\max }
$$

em que:

Zo - cota de cabeceira fíctícia inicial, em $\mathrm{m}$

$\mathrm{CHf}_{\text {max }}$ - carga hidráulica fictícia máxima, em $\mathrm{m}$

De posse da solução inicial, dá-se início à segunda etapa do método, que consiste na execução de um processo iterativo de operações que reduz gradualmente a cota piezométrica de alimentação, com o mínimo acréscimo de custo da rede.

Para determinar a intervenção ou modificação em certa tubulação da rede, que provoque o menor aumento de custo, utilizar-se-á o conceito de Gradiente de Câmbio desenvolvido por Granados (1990). O gradiente de câmbio de determinado 
trecho da rede corresponde ao acréscimo de custo produzido pela substituição de seu diâmetro pelo nominal consecutivo superior, relacionado com a diminuição da perda de carga correspondente e representa o custo marginal da diminuição da perda de carga, alcançada em um trecho determinado, mediante a substituição do seu diâmetro pelo consecutivo superior, e é expresso por:

$$
\mathrm{C}=\frac{\mathrm{P}_{2}-\mathrm{P}_{1}}{\mathrm{hf}_{1}-\mathrm{hf}_{2}}
$$

em que:

C - gradiente de câmbio do trecho, em $\mathrm{R} \$ \mathrm{~m}^{-1}$

$\mathrm{P}_{1}$ - custo total da tubulação do trecho com o diâmetro $\mathrm{D}_{1}$ em R $\$$

$\mathrm{P}_{2}$ - custo total da tubulação do trecho com o diâmetro consecutivo superior $\mathrm{D}_{2}$, em $\mathrm{R} \$$

$\mathrm{hf}_{1}$ - perda de carga no trecho com o diâmetro $\mathrm{D}_{1}$, em m

$\mathrm{hf}_{2}$ - perda de carga no trecho com o diâmetro consecutivo superior $\mathrm{D}_{2}, \mathrm{em} \mathrm{m}$

Para reduzir a cota piezométrica de alimentação da rede é necessário diminuir as perdas de carga que ocorrem nas artérias mais desfavoráveis do sistema de distribuição de água. Em termos de diminuição da perda de carga com o objetivo de baixar a cota de alimentação, é indiferente que essa diminuição se produza em qualquer trecho da artéria mais desfavorável. Como se pretende baixar a cota com o menor acréscimo de custo, a diminuição da perda de carga (aumento do diâmetro da tubulação) deve ocorrer no trecho cuja mudança de diâmetro proporciona menor aumento de custo da rede por unidade de altura de carga. Assim sendo, para se baixar a cota de cabeceira da rede com o menor acréscimo de seu custo, deve-se aumentar o diâmetro do trecho de menor gradiente de câmbio pertencente à artéria mais desfavorável; este trecho, sujeito à modificação, será denominado trecho potencial $\left(\mathrm{T}^{*}\right)$; desta forma, obtém-se uma melhoria na perda de carga, proporcionada pela troca do diâmetro $\left(\mathrm{D}_{1}\right)$ por seu consecutivo superior $\left(\mathrm{D}_{2}\right)$ :

$$
\Delta \mathrm{hf}=\mathrm{hf}_{1}-\mathrm{hf}_{2}
$$

A Figura 1 mostra o esquema de uma rede de distribuição de água com insuficiência de pressão nos pontos de consumo, em três situações distintas: situação do projeto original, na solução inicial do método (rede com cota de cabeceira fictícia inicial) e na solução definitiva (rede com configuração proposta ao final da avaliação). Na situação do projeto original (linha piezométrica tracejada) nota-se que apenas nos nós 1,4 e 8 a pressão disponível atende à requerida devido ao aumento das perdas de carga não previstas no projeto. Na solução inicial do método há acréscimo em toda a linha piezométrica da rede (linha piezométrica pontilhada), de maneira que no nó mais desfavorável a pressão disponível seja igual à requerida; já na solução definitiva (linha piezométrica cheia), a cota de cabeceira final coincide com a cota original do reservatório, em decorrência da substituição de algumas tubulações antigas da rede por novas de maiores diâmetros. Essas substituições são as que acarretam os menores aumentos de custo no sistema, proporcionando a solução ótima da reabilitação.

Em cada iteração da segunda etapa do método, o decréscimo de pressão na cabeceira da rede $(\Delta \mathrm{Z})$ será o menor valor entre a melhora da perda de carga $(\Delta \mathrm{hf})$, no trecho potencial e o mínimo excesso de pressão nos diversos pontos da rede excetuando-se os excessos de pressão dos nós situados à jusante do(s) trecho(s) potencial(is). Caso o valor de $\Delta \mathrm{hf}$ disponível do trecho potencial seja menor que o excesso de pressão mínima na rede $\left(\mathrm{EP}_{\text {min }}\right)$, o decréscimo de pressão na cabeceira será igual ao valor $\Delta \mathrm{hf}$ e o trecho potencial será ocupado pelo diâmetro de maior calibre. Neste caso, a linha piezométrica da rede resultante ficará rebaixada em $\Delta Z$ (excetuando o trecho potencial e aqueles situados à jusante do mesmo). Os novos excessos de pressão de toda a rede serão iguais aos anteriores, menos $\Delta \mathrm{Z}$ ou $\Delta \mathrm{hf}$, Com exceção dos excessos correspondentes ao trecho potencial e aos trechos situados à jusante do mesmo, que permanecem invariáveis.

Quando $\Delta \mathrm{hf}$ disponível do trecho potencial é maior que $\mathrm{EP}_{\text {min }}$, o decréscimo de pressão na cabeceira será igual a $\mathrm{EP}_{\text {min }}$, e o trecho potencial se torna composto por dois diâmetros (o anterior e o consecutivo superior). Em tal caso e para este trecho, o novo valor de $\Delta \mathrm{hf}$ disponível será igual ao $\Delta \mathrm{hf}$ disp

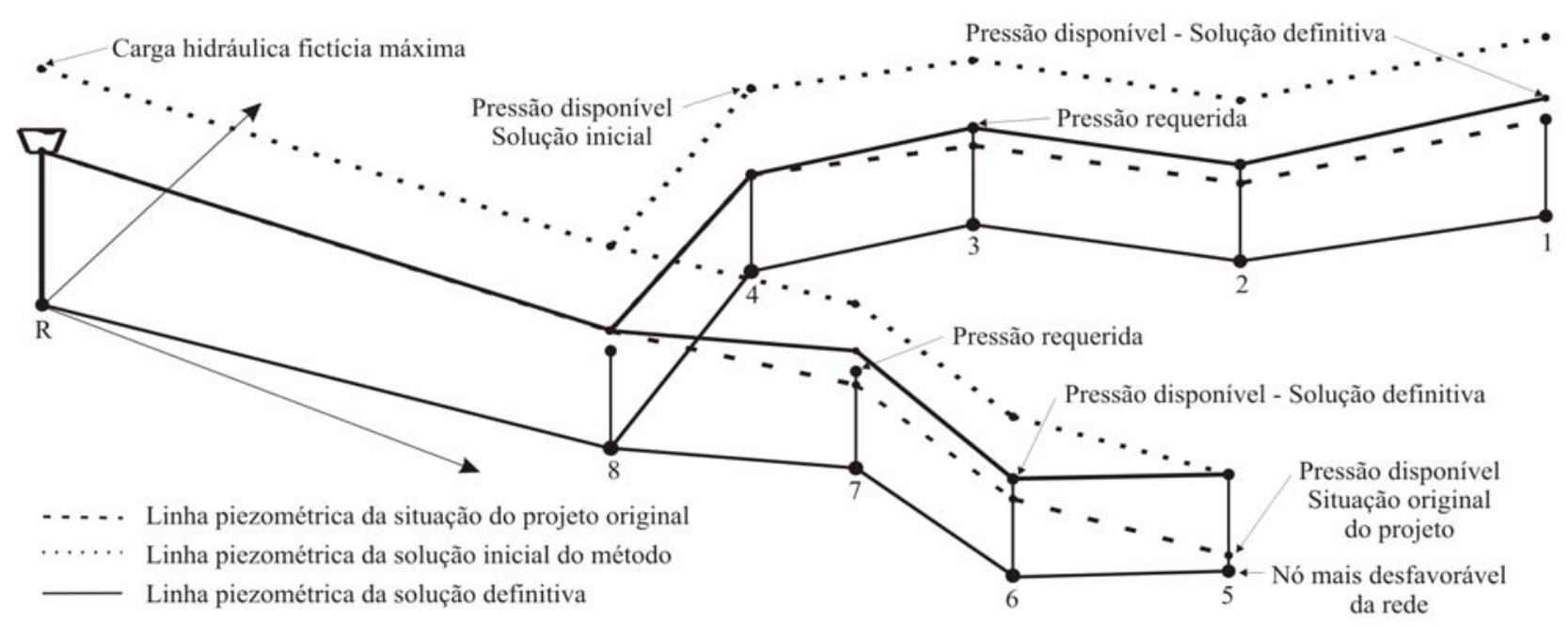

Figura 1. Esquema das linhas piezométricas para as três situações de projeto 
anterior, menos $\Delta \mathrm{Z}$ ou $\mathrm{EP}_{\min }$. A nova cota piezométrica de cabeceira e os novos excessos de pressão da rede ficarão rebaixados em $\mathrm{EP}_{\text {min }}$, com exceção do trecho potencial e dos situados à jusante do mesmo.

Definida a nova cota de cabeceira da rede, faz-se necessário reajustar os preços e gradientes de câmbio das tubulações. O novo custo de reabilitação será igual ao custo anterior de reabilitação mais a diferença de custo proporcionado pelo aumento de diâmetro do(s) trecho(s) potencial(s).

A metodologia da segunda etapa pode ser sintetizada em:

- determinação do gradiente de câmbio ótimo da rede $\left(\mathrm{C}^{*}\right)$;

- cálculo do decréscimo da cota piezométrica de cabeceira $(\Delta \mathrm{Z})$

- modificação dos diâmetros dos trechos ótimos (T*);

- obtenção da nova cota piezométrica de cabeceira e dos novos excessos de pressão;

- reajuste dos custos e dos gradientes de câmbio nos trechos onde houve mudança;

- cálculo do novo custo ótimo de reabilitação da rede.

O método de otimização segue a metodologia antes apresentada, baixando a cota piezométrica de cabeceira, até alcançar a cota piezométrica do reservatório existente.

\section{RESULTADOS E DISCUSSÃO}

O sistema escolhido para a reabilitação com o menor custo possível, foi uma rede fictícia coletiva de distribuição de água, que abastece um projeto de irrigação com parcelas irrigadas por microaspersão, cujo traçado e topografia são mostrados na Figura 2.

A Tabela 1 apresenta os dados da rede e as condições de operação previstas, enquanto a Tabela 2 fornece os preços de implantação das tubulações. Dados:

- pressão mínima requerida nos nós: $\mathrm{P}_{\min }=147,10 \mathrm{KPa}(15 \mathrm{~m})$

- tubulações de PVC, coeficiente de Hazen-Williams: $\mathrm{C}_{\text {novo }}=145, \mathrm{C}_{\text {usado }}=125$

- altura do NA mínimo do reservatório: $\mathrm{H}=25 \mathrm{~m}$

Como se observa na Tabela 1, apenas nos nós 3, 6 e 11 as pressões disponíveis são superiores às requeridas. $\mathrm{O}$ primeiro passo da metodologia consiste na determinação das cargas hidráulicas fíctícias nos nós, cujos valores estão descritos na

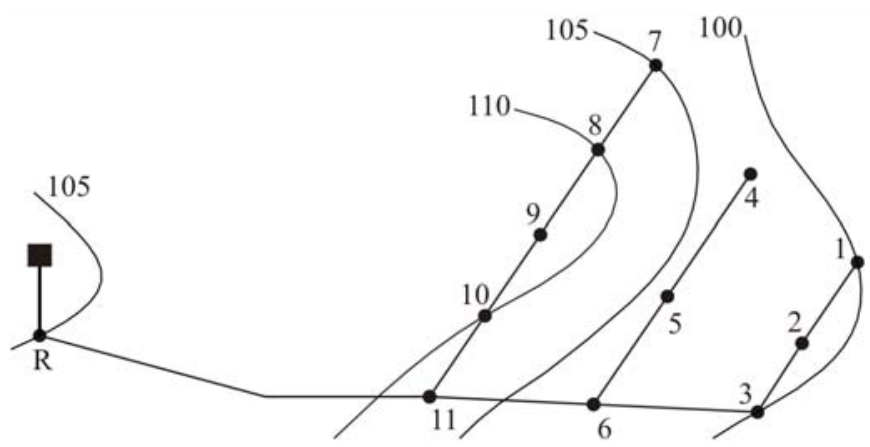

Figura 2. Esquema da rede de distribuição

Tabela 2. Custos de implantação, por metro linear, das tubulações de PVC/DEFoFo

\begin{tabular}{cccc}
\hline $\begin{array}{c}\text { Diâmetro } \\
\text { nominal }\end{array}$ & $\begin{array}{c}\text { Custo da } \\
\text { tubulação } \\
\left(\mathrm{R} \$ \text { m }^{-1}\right)\end{array}$ & $\begin{array}{c}\text { Custo de } \\
\text { montagem } \\
\left(\mathrm{R} \$ \mathrm{~m}^{-1}\right)\end{array}$ & $\begin{array}{c}\text { Custo de } \\
\text { implantação } \\
\left(\mathrm{R} \$ \mathrm{~m}^{-1}\right)\end{array}$ \\
\hline 100 & 17,09 & 24,30 & 41,39 \\
150 & 33,80 & 26,89 & 60,69 \\
200 & 57,62 & 32,78 & 90,40 \\
250 & 88,59 & 34,59 & 123,18 \\
300 & 122,24 & 40,02 & 162,26 \\
400 & 207,09 & 45,64 & 252,73 \\
500 & 319,44 & 53,16 & 372,60 \\
\hline Fonte: CAGEPA - Brasil, 2004 & & \\
\hline
\end{tabular}

Tabela 3. A cota piezométrica de cabeceira inicial será o maior valor entre as cargas hidráulicas fictícias $\left(\mathrm{CHf}_{\max }\right)$, neste caso, $135,47 \mathrm{~m}(\mathrm{Zo}=135,47 \mathrm{~m})$; em seguida, executa-se o processo interativo de cálculo no qual se baixa, passo a passo, a cota piezométrica de cabeceira inicial, até alcançar a cota de $130 \mathrm{~m}$.

Encontram-se, na Tabela 4, os resultados das variáveis necessárias para fornecer, em cada iteração, o custo ótimo da reabilitação da rede de distribuição (CR) e a cota piezométrica de cabeceira $\left(\mathrm{Z}_{\mathrm{i}}\right)$ correspondente. A Tabela ainda fornece, em

Tabela 3. Carga hidráulica fictícia dos nós em metro

\begin{tabular}{cccccccc}
\hline Nó & CHf & Nó & CHf & Nó & CHf & Nó & CHf \\
\hline 1 & 131,2 & 4 & 135,47 & 7 & 130,1 & 10 & 130,3 \\
2 & 131,5 & 5 & 132,1 & 8 & 133,4 & 11 & 112,3 \\
3 & 128,6 & 6 & 127,6 & 9 & 131,3 & & \\
\hline
\end{tabular}

Tabela 1. Situação do projeto existente da rede de distribuição

\begin{tabular}{|c|c|c|c|c|c|c|c|c|c|c|c|c|}
\hline Trecho & $\begin{array}{l}\text { Vazão } \\
\left(\mathrm{L} \mathrm{s}^{-1}\right)\end{array}$ & $\begin{array}{l}\text { DN } \\
(\mathrm{mm})\end{array}$ & $\begin{array}{c}\text { D interno } \\
(\mathrm{mm})\end{array}$ & $\begin{array}{l}\text { Coefic. } \\
\text { H. W. }\end{array}$ & $\begin{array}{l}\text { Comp. } \\
\text { (m) }\end{array}$ & $\begin{array}{l}\text { Perdas } \\
\text { (m) }\end{array}$ & Nó & $\begin{array}{l}\text { Altura } \\
\text { piezométrica } \\
(\mathrm{m})\end{array}$ & $\begin{array}{c}\text { Cota do } \\
\text { terreno } \\
(\mathrm{m})\end{array}$ & $\begin{array}{l}\text { Pressão } \\
\text { requerida } \\
\text { (m) }\end{array}$ & $\begin{array}{c}\text { Pressão } \\
\text { disponível } \\
(\mathrm{m})\end{array}$ & $\begin{array}{c}\text { Déficit de } \\
\text { pressão } \\
(\mathrm{m})\end{array}$ \\
\hline $1-2$ & 6,00 & 100 & 108,4 & 125 & 120 & 0,64 & 1 & 113,83 & 100,0 & 15 & 13,83 & 1,17 \\
\hline $2-3$ & 12,00 & 100 & 108,4 & 125 & 100 & 1,93 & 2 & 114,47 & 101,0 & 15 & 13,47 & 1,53 \\
\hline $3-6$ & 32,00 & 150 & 156,4 & 125 & 200 & 3,99 & 3 & 116,41 & 100,0 & 15 & 16,41 & - \\
\hline $4-5$ & 14,40 & 100 & 108,4 & 125 & 180 & 4,88 & 4 & 112,03 & 102,5 & 15 & 9,53 & 5,47 \\
\hline $5-6$ & 12,80 & 100 & 108,4 & 125 & 160 & 3,49 & 5 & 116,91 & 104,0 & 15 & 12,91 & 2,09 \\
\hline $6-11$ & 75,20 & 200 & 204,2 & 125 & 200 & 5,30 & 6 & 120,40 & 103,0 & 15 & 17,40 & - \\
\hline $7-8$ & 10,00 & 100 & 108,4 & 125 & 125 & 1,72 & 7 & 119,90 & 105,0 & 15 & 14,90 & 0,10 \\
\hline $8-9$ & 20,00 & 150 & 156,4 & 125 & 125 & 1,04 & 8 & 121,63 & 110,0 & 15 & 11,63 & 3,37 \\
\hline $9-10$ & 29,60 & 150 & 156,4 & 125 & 120 & 2,07 & 9 & 122,67 & 109,0 & 15 & 13,67 & 1,33 \\
\hline $10-11$ & 39,20 & 200 & 204,2 & 125 & 120 & 0,95 & 10 & 124,74 & 110,0 & 15 & 14,74 & 0,26 \\
\hline $11-\mathrm{R}$ & 114,40 & 300 & 299,8 & 125 & 485 & 4,31 & 11 & 125,69 & 108,0 & 0 & 17,69 & - \\
\hline
\end{tabular}

Cota piezométrica de cabeceira $=130 \mathrm{~m} ;$ Altura do reservatório $=25 \mathrm{~m}$ 
Tabela 4. Processo de iterações*

\begin{tabular}{|c|c|c|c|c|c|}
\hline Trecho & $\begin{array}{c}\text { Do } \\
(\mathrm{mm})\end{array}$ & $\begin{array}{c}\mathrm{CR} \\
\left(\mathrm{R} \$ \mathrm{~m}^{-1}\right)\end{array}$ & $\begin{array}{l}\text { EP } \\
(\mathrm{m})\end{array}$ & $\begin{array}{l}\Delta \mathrm{hf} \\
(\mathrm{m})\end{array}$ & $\begin{array}{l}\mathrm{Pd} \\
(\mathrm{m})\end{array}$ \\
\hline \multicolumn{2}{|c|}{ Solução inicial } & \multicolumn{4}{|c|}{ Cota piezométrica de cabeceira $(\mathrm{Zo})=135,47 \mathrm{~m}$} \\
\hline $1-2$ & 100 & $12.987,00$ & 4,30 & 0,56 & 19,30 \\
\hline $2-3$ & 100 & $3.597,00$ & 3,94 & 1,69 & 18,94 \\
\hline $3-6$ & 150 & $5.717,00$ & 6,87 & 3,16 & 21,87 \\
\hline$\underline{4-5}$ & 100 & $2.567,00$ & 0,00 & 4,26 & 15,00 \\
\hline $5-6$ & 100 & $3.192,00$ & 3,38 & 3,04 & 18,38 \\
\hline $6-11$ & 200 & $6.394,00$ & 7,86 & 3,85 & 22,86 \\
\hline $7-8$ & 100 & $5.042,00$ & 5,37 & 1,50 & 20,37 \\
\hline $8-9$ & 150 & $13.652,00$ & 2,095 & 0,83 & 17,09 \\
\hline $9-10$ & 150 & $6.605,00$ & 4,14 & 1,64 & 19,14 \\
\hline $10-11$ & 200 & $21.369,00$ & 5,21 & 0,69 & 20,21 \\
\hline $11-\mathrm{R}$ & 300 & $35.553,00$ & 23,16 & 3,45 & 23,16 \\
\hline \multicolumn{2}{|c|}{$1^{\mathrm{a}}$ iteração } & \multicolumn{4}{|c|}{ Cota piezométrica de cabeceira $\left(\mathrm{Z}_{1}\right)=133,37 \mathrm{~m}$} \\
\hline $1-2$ & 100 & $12.987,00$ & 2,20 & 0,56 & 17,20 \\
\hline $2-3$ & 100 & $3.597,00$ & 1,85 & 1,69 & 16,85 \\
\hline $3-6$ & 150 & $5.717,00$ & 4,78 & 3,16 & 19,78 \\
\hline$\underline{4-5}$ & $100 / 150$ & $2.567,00$ & 0,00 & 2,16 & 15,00 \\
\hline $5-6$ & 100 & $3.192,00$ & 1,283 & 3,04 & 16,28 \\
\hline $6-11$ & 200 & $6.394,00$ & 5,77 & 3,85 & 20,77 \\
\hline $7-8$ & 100 & $5.042,00$ & 3,28 & 1,50 & 18,28 \\
\hline $8-9$ & 150 & $13.652,00$ & 0,00 & 0,83 & 15,00 \\
\hline $9-10$ & 150 & $6.605,00$ & 2,04 & 1,64 & 17,04 \\
\hline $10-11$ & 200 & $21.369,00$ & 3,12 & 0,69 & 18,12 \\
\hline $11-\mathrm{R}$ & 300 & $35.553,00$ & 21,07 & 3,45 & 21,07 \\
\hline \multicolumn{2}{|c|}{$2^{\mathrm{a}}$ iteração } & \multicolumn{4}{|c|}{ Cota piezométrica de cabeceira $\left(\mathrm{Z}_{2}\right)=132,09 \mathrm{~m}$} \\
\hline $1-2$ & 100 & $12.987,00$ & 0,92 & 0,56 & 15,92 \\
\hline $2-3$ & 100 & $3.597,00$ & 0,56 & 1,69 & 15,56 \\
\hline $3-6$ & 150 & $5.717,00$ & 3,50 & 3,16 & 18,50 \\
\hline $4-5$ & $100 / 150$ & $2.567,00$ & 0,00 & 0,88 & 15,00 \\
\hline$\underline{5-6}$ & 100 & $3.192,00$ & 0,00 & 3,04 & 15,00 \\
\hline $6-11$ & 200 & $6.394,00$ & 4,49 & 3,85 & 19,49 \\
\hline $7-8$ & 100 & $5.042,00$ & 3,28 & 1,50 & 18,28 \\
\hline $8-9$ & 150 & $13.652,00$ & 0,00 & 0,83 & 15,00 \\
\hline$\underline{9-10}$ & $150 / 200$ & $6.605,00$ & 2,04 & 0,359 & 17,04 \\
\hline $10-11$ & 200 & $21.369,00$ & 1,83 & 0,69 & 16,83 \\
\hline $11-\mathrm{R}$ & 300 & $35.553,00$ & 19,78 & 3,45 & 19,78 \\
\hline \multicolumn{2}{|c|}{$3^{\mathrm{a}}$ iteração } & \multicolumn{4}{|c|}{ Cota piezométrica de cabeceira $\left(Z_{3}\right)=131,73 \mathrm{~m}$} \\
\hline $1-2$ & 100 & $12.987,00$ & 0,56 & 0,56 & 15,56 \\
\hline $2-3$ & 100 & $3.597,00$ & 0,204 & 1,69 & 15,20 \\
\hline $3-6$ & 150 & $5.717,00$ & 3,14 & 3,16 & 18,14 \\
\hline $4-5$ & $100 / 150$ & $2.567,00$ & 0,00 & 0,88 & 15,00 \\
\hline$\underline{5-6}$ & $100 / 150$ & $3.192,00$ & 0,00 & 2,68 & 15,00 \\
\hline$\overline{6-11}$ & 200 & $6.394,00$ & 4,13 & 3,85 & 19,13 \\
\hline $7-8$ & 100 & $5.042,00$ & 3,28 & 1,50 & 18,28 \\
\hline$\underline{8-9}$ & 150 & $13.652,00$ & 0,00 & 0,83 & 15,00 \\
\hline $9-10$ & 200 & $14.290,00$ & 2,04 & 0,28 & 17,04 \\
\hline $10-11$ & 200 & $21.369,00$ & 1,47 & 0,69 & 16,47 \\
\hline $11-\mathrm{R}$ & 300 & $35.553,00$ & 19,42 & 3,45 & 19,42 \\
\hline \multicolumn{2}{|c|}{$4^{\mathrm{a}}$ iteração } & \multicolumn{4}{|c|}{ Cota piezométrica de cabeceira $\left(\mathrm{Z}_{4}\right)=131,53 \mathrm{~m}$} \\
\hline $1-2$ & 100 & $12.987,00$ & 0,36 & 0,56 & 15,36 \\
\hline $2-3$ & 100 & $3.597,00$ & 0,00 & 1,69 & 15,00 \\
\hline $3-6$ & 150 & $5.717,00$ & 2,93 & 3,16 & 17,93 \\
\hline $4-5$ & $100 / 150$ & $2.567,00$ & 0,00 & 0,88 & 15,00 \\
\hline $5-6$ & $100 / 150$ & $3.192,00$ & 0,00 & 2,48 & 15,00 \\
\hline 6-11 & 200 & $6.394,00$ & 3,92 & 3,85 & 18,92 \\
\hline $7-8$ & 100 & $5.042,00$ & 3,28 & 1,50 & 18,28 \\
\hline$\underline{8-9}$ & $150 / 200$ & $13.652,00$ & 0,00 & 0,624 & 15,00 \\
\hline $9-10$ & 200 & $14.290,00$ & 1,84 & 0,28 & 16,84 \\
\hline $10-11$ & 200 & $21.369,00$ & 1,27 & 0,69 & 16,27 \\
\hline $11-\mathrm{R}$ & 300 & $35.553,00$ & 19,22 & 3,45 & 19,22 \\
\hline
\end{tabular}

Continua...
Continuação - Tabela 4.

\begin{tabular}{|c|c|c|c|c|c|}
\hline Trecho & $\begin{array}{c}\text { Do } \\
(\mathrm{mm})\end{array}$ & $\begin{array}{c}\mathrm{CR} \\
\left(\mathrm{R} \$ \mathrm{~m}^{-1}\right)\end{array}$ & $\begin{array}{l}\text { EP } \\
(\mathrm{m})\end{array}$ & $\begin{array}{l}\Delta \mathrm{hf} \\
(\mathrm{m})\end{array}$ & $\begin{array}{l}\mathrm{Pd} \\
(\mathrm{m})\end{array}$ \\
\hline \multicolumn{2}{|c|}{$5^{\mathrm{a}}$ iteração } & \multicolumn{4}{|c|}{ Cota piezométrica de cabeceira $\left(Z_{5}\right)=130,90 \mathrm{~m}$} \\
\hline $1-2$ & 100 & $12.987,00$ & 0,36 & 0,56 & 15,36 \\
\hline $2-3$ & 100 & $3.597,00$ & 0,00 & 1,69 & 15,00 \\
\hline $3-6$ & 150 & $5.717,00$ & 2,93 & 3,16 & 17,93 \\
\hline $4-5$ & $100 / 150$ & $2.567,00$ & 0,00 & 0,88 & 15,00 \\
\hline $5-6$ & $100 / 150$ & $3.192,00$ & 0,00 & 2,48 & 15,00 \\
\hline$\underline{6-11}$ & $200 / 250$ & $6.394,00$ & 3,92 & 3,23 & 18,92 \\
\hline$\overline{7-8}$ & 100 & $5.042,00$ & 3,28 & 1,50 & 18,28 \\
\hline $8-9$ & 200 & $29.535,00$ & 0,00 & 0,14 & 15,00 \\
\hline $9-10$ & 200 & $14.290,00$ & 1,22 & 0,275 & 16,22 \\
\hline$\overline{10-11}$ & 200 & $21.369,00$ & 0,65 & 0,69 & 15,65 \\
\hline $11-\mathrm{R}$ & 300 & $35.553,00$ & 18,60 & 3,45 & 18,60 \\
\hline \multicolumn{2}{|c|}{$6^{\mathrm{a}}$ iteração } & \multicolumn{4}{|c|}{ Cota piezométrica de cabeceira $\left(\mathrm{Z}_{6}\right)=130,63 \mathrm{~m}$} \\
\hline $1-2$ & 100 & $12.987,00$ & 0,36 & 0,56 & 15,36 \\
\hline $2-3$ & 100 & $3.597,00$ & 0,00 & 1,69 & 15,00 \\
\hline $3-6$ & 150 & $5.717,00$ & 2,93 & 3,16 & 17,93 \\
\hline $4-5$ & $100 / 150$ & $2.567,00$ & 0,00 & 0,88 & 15,00 \\
\hline $5-6$ & $100 / 150$ & $3.192,00$ & 0,00 & 2,48 & 15,00 \\
\hline$\underline{6-11}$ & $200 / 250$ & $6.394,00$ & 3,92 & 2,95 & 18,92 \\
\hline $7-8$ & 100 & $5.042,00$ & 3,28 & 1,50 & 18,28 \\
\hline $8-9$ & 200 & $29.535,00$ & 0,00 & 0,14 & 15,00 \\
\hline $9-10$ & 250 & $53.278,00$ & 1,22 & 0,09 & 16,22 \\
\hline$\underline{10-11}$ & 200 & $21.369,00$ & 0,37 & 0,69 & 15,37 \\
\hline $11-\mathrm{R}$ & 300 & $35.553,00$ & 18,32 & 3,45 & 18,32 \\
\hline \multicolumn{2}{|c|}{$7^{\mathrm{a}}$ iteração } & \multicolumn{4}{|c|}{ Cota piezométrica de cabeceira $\left(\mathrm{Z}_{7}\right)=130 \mathrm{~m}$} \\
\hline $1-2$ & 100 & $12.987,00$ & 0,36 & 0,56 & 15,36 \\
\hline $2-3$ & 100 & $3.597,00$ & 0,00 & 1,69 & 15,00 \\
\hline $3-6$ & 150 & $5.717,00$ & 2,93 & 3,16 & 17,93 \\
\hline $4-5$ & $100 / 150$ & $2.567,00$ & 0,00 & 0,88 & 15,00 \\
\hline $5-6$ & $100 / 150$ & $3.192,00$ & 0,00 & 2,48 & 15,00 \\
\hline $6-11$ & $200 / 250$ & $6.394,00$ & 3,92 & 2,33 & 18,92 \\
\hline $7-8$ & 100 & $5.042,00$ & 3,28 & 1,50 & 18,28 \\
\hline $8-9$ & 200 & $29.535,00$ & 0,00 & 0,14 & 15,00 \\
\hline $9-10$ & 250 & $53.278,00$ & 1,22 & 0,09 & 16,22 \\
\hline $10-11$ & $200 / 250$ & $21.369,00$ & 0,37 & 0,06 & 15,37 \\
\hline $11-\mathrm{R}$ & 300 & $35.553,00$ & 17,69 & 3,45 & 17,69 \\
\hline
\end{tabular}

cada iteração, para o(s) diâmetro(s) de cada trecho, o gradiente de câmbio (coluna 3 ), o excesso de pressão (coluna 4), a perda de carga disponível (coluna 5) e a pressão disponível no nó de jusante de cada trecho (coluna 6).

Na solução inicial, o $\mathrm{EP}=0$ ocorre no nó 4 , o menor gradiente de câmbio na artéria formada pelos trechos $4,5,6$ e 11 corresponde ao do trecho 4 , que é igual a $2.567,00 \mathrm{R} \$ \mathrm{~m}^{-1}$. O decréscimo da cota de cabeceira será igual a $2,09 \mathrm{~m}$, referente ao menor valor entre a perda de carga disponível no trecho $4 \mathrm{e}$ os excessos de pressão nos outros trechos da rede. O custo de reabilitação da rede será igual a $\mathrm{R} \$ 5.377,00$, que é o resultado do produto de $\Delta \mathrm{hf}_{8}$ por $\mathrm{C}^{*}$; com isto, o trecho será ocupado pelos diâmetros 100 e 150 e os novos excessos de pressão passam a ser iguais aos anteriores menos 2,09 m. Os valores das pressões disponíveis nos nós serão iguais, em cada iteração, aos valores de EP mais a pressão requerida.

Como resultado da $1^{\mathrm{a}}$ iteração os excessos de pressão são nulos nos nós 1 e 3 , situados em paralelo; como conseqüência, o gradiente de câmbio ótimo será o menor valor entre os diversos valores resultantes da soma dos gradientes de câmbios dos trechos em paralelo das artérias mais desfavoráveis: $\mathrm{C}_{4}+\mathrm{C}_{6}$ $\left(6.219,00 \mathrm{R} \$ \mathrm{~m}^{-1}\right), \mathrm{C}_{4}+\mathrm{C}_{9}\left(9.172,00 \mathrm{R} \$ \mathrm{~m}^{-1}\right), \mathrm{C}_{4}+\mathrm{C}_{10}(23.936,00 \mathrm{R} \$$ 
$\left.\mathrm{m}^{-1}\right), \mathrm{C}_{5}+\mathrm{C}_{8}\left(16.844,00 \mathrm{R} \$ \mathrm{~m}^{-1}\right), \mathrm{C}_{5}+\mathrm{C}_{9}\left(9.797,00 \mathrm{R} \$ \mathrm{~m}^{-1}\right), \mathrm{C}_{5}+\mathrm{C}_{10}$ $\left(24.561,00 \mathrm{R} \$ \mathrm{~m}^{-1}\right), \mathrm{C}_{6}+\mathrm{C}_{8}\left(20.047,00 \mathrm{R} \$ \mathrm{~m}^{-1}\right), \mathrm{C}_{6}+\mathrm{C}_{9}(12.999,00 \mathrm{R} \$$ $\left.\mathrm{m}^{-1}\right) \mathrm{e} \mathrm{C}_{6}+\mathrm{C}_{10}\left(27.763,00 \mathrm{R} \$ \mathrm{~m}^{-1}\right)$; neste caso, $\mathrm{C}^{*}=\mathrm{C}_{4}+\mathrm{C}_{9}(9.172,00$ $\left.\mathrm{R} \$ \mathrm{~m}^{-1}\right)$. O decréscimo de pressão na cota de cabeceira $(\Delta \mathrm{Z})$ será o menor valor entre a melhora da perda de carga $\left(\Delta \mathrm{hf}_{4} \mathrm{e}\right.$ $\Delta \mathrm{hf}_{9}$ ) nos trechos potenciais (trechos $4 \mathrm{e}$ 9), e o mínimo excesso de pressão nos diversos pontos da rede situados à jusante dos trechos potencias $\left(\mathrm{EP}_{1}, \mathrm{EP}_{2}, \mathrm{EP}_{3}, \mathrm{EP}_{5}, \mathrm{EP}_{6}, \mathrm{EP}_{10} \mathrm{e} \mathrm{EP}_{11}\right)$; portanto, o decréscimo da cota de cabeceira nesta iteração será igual a $1,28 \mathrm{~m}$.

O processo iterativo do método de otimização segue até alcançar a cota desejada de $130 \mathrm{~m}$, quando finaliza a reabilitação ótima da rede. Os valores das variáveis da última iteração $\left(7^{a}\right)$ sintetizam os resultados cujo custo de recuperação é igual a $\mathrm{R} \$ 59.723,00$. Os diâmetros definitivos dos trechos e as pressões disponíveis resultantes, se encontram nas colunas 2 e 6 , respectivamente. As combinações para o cálculo do gradiente de câmbio estão descritas resumidamente, na Tabela 5 .

Tabela 5. Resumo do cálculo dos gradientes de câmbio $\left(\mathrm{R} \$ \mathrm{~m}^{-1}\right)$

\begin{tabular}{|c|c|c|c|}
\hline \multicolumn{3}{|c|}{$\mathrm{R} \$$} & $\mathrm{R} \$$ \\
\hline \multicolumn{2}{|c|}{$1^{\mathrm{a}}$ iteração } & \multicolumn{2}{|c|}{$2^{\mathrm{a}}$ iteração } \\
\hline $\mathrm{C}_{4}$ & $2.567,00$ & $\mathrm{C}_{4}+\mathrm{C}_{8}$ & $16.219,00$ \\
\hline $\mathrm{C}_{5}$ & $3.192,00$ & $\mathrm{C}_{4}+\mathrm{C}_{9}$ & $9.172,00$ \\
\hline $\mathrm{C}_{6}$ & $6.394,00$ & $\mathrm{C}_{4}+\mathrm{C}_{10}$ & $23.936,00$ \\
\hline \multirow{7}{*}{$\mathrm{C}_{11}$} & $35.553,00$ & $\mathrm{C}_{5}+\mathrm{C}_{8}$ & $16.844,00$ \\
\hline & & $\mathrm{C}_{5}+\mathrm{C}_{9}$ & $9.797,00$ \\
\hline & & $\mathrm{C}_{5}+\mathrm{C}_{10}$ & $24.561,00$ \\
\hline & & $\mathrm{C}_{6}+\mathrm{C}_{8}$ & $20.047,00$ \\
\hline & & $\mathrm{C}_{6}+\mathrm{C}_{9}$ & $12.999,00$ \\
\hline & & $\mathrm{C}_{6}+\mathrm{C}_{9}$ & $27.763,00$ \\
\hline & & $\mathrm{C}_{11}$ & $35.553,00$ \\
\hline \multicolumn{2}{|c|}{$3^{\mathrm{a}}$ iteração } & \multicolumn{2}{|c|}{$4^{\mathrm{a}}$ iteração } \\
\hline $\mathrm{C}_{5}+\mathrm{C}_{8}$ & $16.844,00$ & $\mathrm{C}_{5}+\mathrm{C}_{8}$ & $16.844,00$ \\
\hline $\mathrm{C}_{5}+\mathrm{C}_{9}$ & $9.797,00$ & $\mathrm{C}_{5}+\mathrm{C}_{9}$ & $17.482,00$ \\
\hline $\mathrm{C}_{5}+\mathrm{C}_{10}$ & $24.561,00$ & $\mathrm{C}_{5}+\mathrm{C}_{10}$ & $24.561,00$ \\
\hline $\mathrm{C}_{6}+\mathrm{C}_{8}$ & $20.047,00$ & $\mathrm{C}_{6}+\mathrm{C}_{8}$ & $20.047,00$ \\
\hline $\mathrm{C}_{6}+\mathrm{C}_{9}$ & $12.999,00$ & $\mathrm{C}_{6}+\mathrm{C}_{9}$ & $20.684,00$ \\
\hline $\mathrm{C}_{6}+\mathrm{C}_{10}$ & $27.763,00$ & $\mathrm{C}_{6}+\mathrm{C}_{10}$ & $27.763,00$ \\
\hline $\mathrm{C}_{11}$ & $35.553,00$ & $\mathrm{C}_{11}$ & $35.553,00$ \\
\hline \multicolumn{2}{|c|}{$5^{\mathrm{a}}$ iteração } & \multicolumn{2}{|c|}{$6^{\mathrm{a}}$ iteração } \\
\hline $\mathrm{C}_{1}+\mathrm{C}_{5}+\mathrm{C}_{8}$ & $29.831,00$ & $\mathrm{C}_{1}+\mathrm{C}_{5}+\mathrm{C}_{8}$ & $45.714,00$ \\
\hline $\mathrm{C}_{1}+\mathrm{C}_{5}+\mathrm{C}_{9}$ & $30.468,00$ & $\mathrm{C}_{1}+\mathrm{C}_{5}+\mathrm{C}_{9}$ & $30.468,00$ \\
\hline $\mathrm{C}_{1}+\mathrm{C}_{5}+\mathrm{C}_{10}$ & $37.548,00$ & $\mathrm{C}_{1}+\mathrm{C}_{5}+\mathrm{C}_{10}$ & $37.548,00$ \\
\hline $\mathrm{C}_{2}+\mathrm{C}_{5}+\mathrm{C}_{8}$ & $20.442,00$ & $\mathrm{C}_{2}+\mathrm{C}_{5}+\mathrm{C}_{8}$ & $36.325,00$ \\
\hline $\mathrm{C}_{2}+\mathrm{C}_{5}+\mathrm{C}_{9}$ & $21.079,00$ & $\mathrm{C}_{2}+\mathrm{C}_{5}+\mathrm{C}_{9}$ & $21.079,00$ \\
\hline $\mathrm{C}_{2}+\mathrm{C}_{5}+\mathrm{C}_{10}$ & $28.159,00$ & $\mathrm{C}_{2}+\mathrm{C}_{5}+\mathrm{C}_{10}$ & $28.159,00$ \\
\hline $\mathrm{C}_{3}+\mathrm{C}_{5}+\mathrm{C}_{8}$ & $22.561,00$ & $\mathrm{C}_{3}+\mathrm{C}_{5}+\mathrm{C}_{8}$ & $38.445,00$ \\
\hline $\mathrm{C}_{3}+\mathrm{C}_{5}+\mathrm{C}_{9}$ & $23.199,00$ & $\mathrm{C}_{3}+\mathrm{C}_{5}+\mathrm{C}_{9}$ & $23.199,00$ \\
\hline $\mathrm{C}_{3}+\mathrm{C}_{5}+\mathrm{C}_{10}$ & $30.278,00$ & $\mathrm{C}_{3}+\mathrm{C}_{5}+\mathrm{C}_{10}$ & $30.278,00$ \\
\hline $\mathrm{C}_{6}+\mathrm{C}_{8}$ & $20.047,00$ & $\mathrm{C}_{6}+\mathrm{C}_{8}$ & $35.930,00$ \\
\hline $\mathrm{C}_{6}+\mathrm{C}_{9}$ & $20.684,00$ & $\mathrm{C}_{6}+\mathrm{C}_{9}$ & $20.684,00$ \\
\hline $\mathrm{C}_{6}+\mathrm{C}_{10}$ & $27.763,00$ & $\mathrm{C}_{6}+\mathrm{C}_{10}$ & $27.763,00$ \\
\hline $\mathrm{C}_{11}$ & $35.553,00$ & $\mathrm{C}_{11}$ & $35.553,00$ \\
\hline \multicolumn{4}{|c|}{$7^{\mathrm{a}}$ iteração } \\
\hline $\mathrm{C}_{1}+\mathrm{C}_{5}+\mathrm{C}_{8}$ & $45.714,00$ & $\mathrm{C}_{3}+\mathrm{C}_{5}+\mathrm{C}_{9}$ & $62.187,00$ \\
\hline $\mathrm{C}_{1}+\mathrm{C}_{5}+\mathrm{C}_{9}$ & $69.457,00$ & $\mathrm{C}_{3}+\mathrm{C}_{5}+\mathrm{C}_{10}$ & $30.278,00$ \\
\hline $\mathrm{C}_{1}+\mathrm{C}_{5}+\mathrm{C}_{10}$ & $37.548,00$ & $\mathrm{C}_{6}+\mathrm{C}_{8}$ & $35.930,00$ \\
\hline $\mathrm{C}_{2}+\mathrm{C}_{5}+\mathrm{C}_{8}$ & $36.325,00$ & $\mathrm{C}_{6}+\mathrm{C}_{9}$ & $59.672,00$ \\
\hline $\mathrm{C}_{2}+\mathrm{C}_{5}+\mathrm{C}_{9}$ & $60.068,00$ & $\mathrm{C}_{6}+\mathrm{C}_{10}$ & $27.763,00$ \\
\hline $\mathrm{C}_{2}+\mathrm{C}_{5}+\mathrm{C}_{10}$ & $28.159,00$ & $\mathrm{C}_{11}$ & $35.553,00$ \\
\hline $\mathrm{C}_{3}+\mathrm{C}_{5}+\mathrm{C}_{8}$ & $38.445,00$ & & \\
\hline
\end{tabular}

Os comprimentos correspondentes aos pares de diâmetro dos trechos 4, 5, 6 e 10, são descritos na Tabela 6 e proporcionais às relações existentes, respectivamente, entre as perdas de carga em cada sub-trecho e a requerida em todo o trecho.

A Tabela 7 apresenta as ações a serem implantadas para a retomada das condições hidráulicas exigidas no projeto da rede de distribuição.

De acordo com os resultados obtidos do método (Tabela 4), o custo de reabilitação da rede deste exemplo é de R $\$$ $59.723,00$, que corresponde a $41 \%$ do custo total de implantação da rede original $(\mathrm{R} \$ 144.032,00)$, calculado com base nos preços constantes na Tabela 2.

Tabela 6. Comprimento dos pares de diâmetros dos trechos 4 , 5,6 e 10

\begin{tabular}{|c|c|c|c|c|c|c|c|c|}
\hline \multirow{2}{*}{ Trecho } & \multicolumn{3}{|c|}{ Sub-trecho 1} & \multicolumn{3}{|c|}{ Sub-trecho 2} & \multicolumn{2}{|c|}{ Trecho } \\
\hline & $\mathrm{D}_{1}$ & $\mathrm{~L}_{1}$ & $h_{f 1}$ & $\mathrm{D}_{2}$ & $\mathrm{~L}_{2}$ & $h_{\mathrm{f} 2}$ & $\mathrm{~L}$ & hf \\
\hline 4 & 100 & 37,15 & 1,01 & 150 & 142,85 & 0,49 & 180 & 1,50 \\
\hline 5 & 100 & 130,37 & 2,84 & 150 & 29,63 & 0,08 & 160 & 2,92 \\
\hline 6 & 200 & 120,76 & 3,20 & 250 & 79,24 & 0,57 & 200 & 3,77 \\
\hline 10 & 200 & 11,12 & 0,09 & 250 & 108,88 & 0,24 & 120 & 0,32 \\
\hline
\end{tabular}

Tabela 7. Decisões de reabilitação propostas pelo método

\begin{tabular}{cccc}
\hline \multirow{2}{*}{ Trecho } & $\begin{array}{c}\text { DN Inicial } \\
(\mathrm{mm})\end{array}$ & \multicolumn{2}{c}{ Solução Proposta } \\
\cline { 3 - 4 } & & DN final $(\mathrm{mm})$ & Comprimento $(\mathrm{m})$ \\
\hline $1-2$ & 100 & 100 & 120 \\
$2-3$ & 100 & 100 & 100 \\
$3-6$ & 150 & 150 & 200 \\
$4-5$ & 100 & 100 & 37 \\
& & 150 & 143 \\
$5-6$ & 100 & 100 & 130 \\
& & 150 & 30 \\
$6-11$ & 200 & 200 & 121 \\
$7-8$ & & 250 & 79 \\
$8-9$ & 100 & 100 & 125 \\
$9-10$ & 150 & 200 & 125 \\
$10-11$ & 150 & 250 & 120 \\
$11-\mathrm{R}$ & 200 & 200 & 11 \\
& & 250 & 485 \\
\hline
\end{tabular}

As decisões sobre as modificações nos trechos da rede foram tomadas com base na minimização dos custos referentes às mudanças nos diâmetros das tubulações, considerando-se que não haveria nenhum inconveniente na intervenção, em determinados trechos da rede. Este inconveniente ocorre em muitas redes, principalmente naquelas situadas em certos centros urbanos; para superá-lo, o projetista deve incorporar um custo marginal referente ao inconveniente provocado pela mudança de diâmetro de alguns trechos da rede.

\section{CONCLUSÕES}

1. A adaptação do método Granados indica uma eficiente alternativa para a tomada de decisão sobre a reabilitação de redes pressurizadas ramificadas, que se encontram com deficiência de vazão e pressão nos pontos de consumo, de 
maneira que a solução encontrada proporcione o menor custo possível de investimento.

2. O método permite, ao analista, visualizar, a cada passo, as diversas alternativas de modificações do sistema original, com vistas à sua reabilitação econômica, podendo ser aplicado diretamente a redes ramificadas de abastecimento urbano e rural, de combate a incêndio, e sistemas pressurizados de irrigação; poderá, ainda, ser empregado em redes malhadas, desde que se utilize o método do seccionamento fictício, para transformá-las em ramificadas.

3. O custo de reabilitação da rede do exemplo deste trabalho foi de $\mathrm{R} \$ 59.723,00$, que corresponde a $41 \%$ do custo de implantação da rede original ( $\mathrm{R} \$ 144.032,00)$.

\section{LITERATURA CITADA}

CAGEPA - Companhia de Água e Esgoto da Paraíba. João Pessoa, 2004. 112p. Relatório Técnico

Cheung, P.B. Análise de reabilitação de redes de distribuição de água para abastecimento via algoritmos genéticos multiobjetivo. 2004. São Carlos: EESC/USP, 2004. 268p. Tese Doutorado

Dandy, G.C.; Engelhardt, M.O. Optimal scheduling of water pipe replacement using genetic algorithms. Journal of Water Resources Planning and Management, Reston: ASCE, v.127, n.4, p.214-223, 2001.

De Schanetzen, W.; Randall-Smith, M.J.; Savic, D.; Walters, G.A. A genetic algorithm approach for rehabilitation in water supply systems. Proceedings of the International Conference Rehabilitation Technology for the Water Industry, Society of British Water Industries, Lille, p.62-83, 1998.

Granados, A. Infraestructuras de regadios: redes colectivas de riego a presión. Madrid: Servicio de Publicación de Escuela Técnica de Ingenieros de Caminos de la Universidad Politécnica de Madrid, 1990. 255p.
Halhal, D.; Walters, G.A.; Ouazar, D.; Savic, D.A. Water network rehabilitation with structured messy genetic algorithm. Journal of Water Resources Planning and Management, Reston: ASCE, v.123, n.3, p.137-146, 1997.

Kim, J.H.; Mays, L.W. Optimal rehabilitation model for water distribution systems. Journal of Water Resource Planning and Management, Reston: ASCE, v.120, n.5, p.674-692, 1994.

Kleiner, Y.; Adams, B.J.; Rogers, J.S. Long-term planning methodology for water distribution system rehabilitation. Water Resources Research, Washington, v.34, n.8, p.20392052, 1998.

Lam, C.F. Discrete gradient optimization of water systems. Journal of Hydraulics Division, Reston, v.99, n.6, p.863-872, 1973.

Le Gat, Y.; Einsenbeis, P. Using maintenance records to forecast failures in water networks. Urban Water, London, v.2, p.173$191,2000$.

Li, D.; Haimes, Y. Optimal maintenance-related decision making deteriorating water distribution systems. Water Resources Research, Washington, v.28, n.4, p.1053-1061, 1992.

Murphy, L.J.; Simpson, A.R. Pipe optimization using genetic algorithms. Research Report No. 93, Adelaide: Department of Civil Engineering, University of Adelaide, 1992. p.95.

Shamir, U.; Howard, C.D.D. An analytic approach to scheduling pipe replacement. Journal of the American Water Works Association. Denver, v.71, n.5, p.248-258, 1979.

Swamee, P.K.; Sharma, A.K. Reorganization of water distribution system. Journal of Environmental Engineering, Reston, v.116, n.3, p.588-600, 1990.

Walski, T.M.; Pelliccia, A. Economic analysis of water main breaks. Journal of the American Water Works Association. Denver, v.74, n.3, p.140-147, 1982.

Walters, G.A.; Halhal, D.; Savic, D.; Ouazar, D. Improved design of Anytown distribution network using structured messy genetic algorithms. Urban Water, London, v.1, p.23-38, 1999. 